\title{
Quicksort: Combining Concurrency, Recursion, and Mutable Data Structures*
}

David Kitchin, Adrian Quark and Jayadev Misra

\begin{abstract}
Quicksort [5] remains one of the most studied algorithms in computer science. It is important not only as a practical sorting method, but also as a splendid teaching aid for introducing recursion and systematic algorithm development. The algorithm has been studied extensively; so, it is natural to assume that everything that needs to be said about it has already been said. Yet, in attempting to code it using a recent programming language of our design, we discovered that its structure is more clearly expressed as a concurrent program that manipulates a shared mutable store, without any locking or explicit synchronization. In this paper, we describe the essential aspects of our programming language Orc [8], show a number of examples that combine its features in various forms, and then develop a concise description of Quicksort. We hope to highlight the importance of including concurrency, recursion and mutability within a single theory.
\end{abstract}

\section{Introduction}

Quicksort [5] remains one of the most studied algorithms in computer science. Its performance has been studied extensively, by Knuth [11] and Sedgewick [17] in particular. A variety of implementations exist on different architectures, and many variants of Quicksort have been developed that improve its performance for specific platforms.

David Kitchin

University of Texas at Austin, e-mail: dkitchin@cs.utexas.edu

Adrian Quark

University of Texas at Austin, e-mail: quark@cs.utexas.edu

Jayadev Misra

University of Texas at Austin, e-mail: misra@cs.utexas.edu

* This work is partially supported by National Science Foundation grant CCF-0811536. 
The structure of the algorithm has also been studied extensively. It presents three important ideas in computing - mutable store, recursion and concurrency- making it attractive as a teaching tool. These aspects cross the usual boundaries of programming languages: pure functional programs typically do not admit in-situ permutation of data elements, imperative programs are typically sequential and do not highlight concurrency, and typical concurrency constructs do not combine well with recursion.

We have recently designed a process calculus [14] and a programming language based on it, called Orc [8]. We believe that the Orc coding of Quicksort, in Section 5, highlights all three of these aspects while remaining faithful to the original intent of the algorithm.

This paper first presents Orc, starting with the Orc calculus, and then the programming language designed around the calculus. The calculus starts with the premise that concurrency is fundamental; sequential programming is a special case. The calculus itself is extremely small, consisting of four combinators (only three of which are essential for this paper) and a definition mechanism. It contains no data structuring, nor any notion of process, thread or communication.

The calculus is next enhanced with a small functional language to ease writing of practical programs. The language includes basic operators, conditionals, some primitive data types, and pattern matching mechanisms. The enhancements are mere syntactic sugar; they can all be translated to the core Orc calculus, and that is how they are actually implemented. The programming model draws its power from external services, called sites, which may encode functionalities that are better expressed in other programming paradigms. The combinators allow these sites to be integrated into a full concurrent program.

The paper is structured as follows. In Section 2, we review the Orc concurrency calculus. Section 3 shows its expansion into a functional concurrent programming language, with a library of sites supporting time, mutable state, communication, and synchronization. Section 4 present a series of example programs using concurrency, recursion, and the additional capabilities provided by the site library. In Section 5, we present the Quicksort algorithm in Orc. Section 6 includes brief concluding remarks.

For a more thorough review of the Orc language, see [8], from which Sections 2, 3, and 4 borrow substantially. We also encourage the reader to visit our website [16]; it hosts a comprehensive user guide [9], a community wiki, and a web-based interface for experimenting with Orc.

\section{The Orc Concurrency Calculus}

The Orc calculus is based on the execution of expressions. Expressions are built up recursively using Orc's concurrency combinators. When executed, an Orc expression invokes services and may publish values. Different executions of the same expression may have completely different behaviors; they may call different services, 
receive different responses from the same service, and publish different values. An expression is silent if it never publishes a value.

In order to invoke services, Orc expressions call sites. A site may be implemented on the client's machine or a remote machine. A site may provide any service; it could run sequential code, transform data, communicate with a web service, or be a proxy for interaction with a human user.

We describe three of the four concurrency combinators of Orc in this paper. Notable omissions in this paper are treatments of logical time (using site Ltimer) and halting (using the fourth concurrency combinator ;). The operational and denotational semantics of the calculus appear in [7].

\subsection{Site Calls}

The simplest Orc expression is a site call $M(\bar{p})$, where $M$ is a site name and $\bar{p}$ is a list of parameters, which are values or variables. The execution of a site call invokes the service associated with $M$, sending it the parameters $\bar{p}$. If the site responds, the call publishes that response. A site responds with at most one value.

Here are some examples of site calls.

add $(3,4) \quad$ Add the numbers 3 and 4 .

CNN (d) Get the CNN news headlines for date d.

Prompt ("Name:") Prompt the user to enter a name on the console.

random (10) Generate a random integer in the range 0..9.

email $(a, m) \quad$ Send message $m$ to email address a

\section{Fundamental Sites}

Though the Orc calculus itself contains no sites, there are a few fundamental sites that are so essential to writing useful programs that we always assume they are available. The site let is the identity site; when passed one argument, it publishes that argument, and when passed multiple arguments it publishes them as a tuple. The site if responds with a signal (a value which carries no information) if its argument is true, and otherwise it does not respond. The site call Rtimer( $t$ ) responds with a signal after exactly t time units.

signal and stop

For convenience, we allow two additional expressions: signal and stop. The expression signal just publishes a signal when executed; it is equivalent to if ( $t$ rue) . The expression stop is simply silent; it is equivalent to if (false). 


\subsection{Combinators}

Orc has four combinators to compose expressions: the parallel combinator $\mid$, the sequential combinator $>x>$, the pruning combinator ${ }^{2}<x<$, and the otherwise combinator $;$. We discuss only the first three in this paper; see [8] for more information on the otherwise combinator.

When composing expressions, the $>x>$ combinator has the highest precedence, followed by $\mid$, then $<x<$.

\section{Parallel Combinator}

In $F \mid G$, expressions $F$ and $G$ execute independently. The sites called by $F$ and $G$ are the ones called by $F \quad G$ and any value published by either $F$ or $G$ is published by $F \mid G$. There is no direct communication or interaction between $F$ and $G$.

For example, evaluation of $\mathrm{CNN}(\mathrm{d}) \quad \mathrm{BBC}(\mathrm{d})$ initiates two independent computations; up to two values will be published depending on the number of responses received.

The parallel combinator is commutative and associative.

\section{Sequential Combinator}

In $F>\mathrm{x}>G$, expression $F$ is first evaluated. Each value published by $F$ initiates a separate execution of $G$ wherein x is bound to that published value. Execution of $F$ continues in parallel with these executions of $G$. If $F$ publishes no value, no execution of $G$ occurs. The values published by the executions of $G$ are the values published by $F>x>G$. The values published by $F$ are consumed.

As an example, the following expression calls sites $\mathrm{CNN}$ and $\mathrm{BBC}$ in parallel to get the news for date $\mathrm{d}$. Responses from either of these calls are bound to $\mathrm{x}$ and then site email is called to send the information to address a. Thus, email may be called 0 , 1 or 2 times, depending on the number of responses received.

$(\operatorname{CNN}(d) \mid \operatorname{BBC}(d))>x>\operatorname{email}(a, x)$

The sequential combinator is right associative, i.e. $F>\mathrm{x}>G>\mathrm{y}>H$ is $F>\mathrm{x}>(G>\mathrm{y}>H)$. When $\mathrm{x}$ is not used in $G$, one may use the short-hand $F>G$ for $F>$ x $>G$.

The sequential combinator generalizes the sequential composition of the traditional imperative languages for a concurrent world: if $F$ publishes a single value and does nothing further, then $F>G$ behaves like an imperative sequential program, $F$ followed by $G$.

\section{Pruning Combinator}

In $F<\mathrm{x}<G$, both $F$ and $G$ execute in parallel. Execution of parts of $F$ that do not depend on $x$ can proceed, but site calls in $F$ for which $x$ is a parameter are suspended

\footnotetext{
${ }^{2}$ In previous publications, $F<\mathrm{x}<G$ was written as $F$ where $x: \in G$.
} 
until $\mathrm{x}$ has a value. If $G$ publishes a value, then $\mathrm{x}$ is assigned that value, $G$ 's execution is terminated and the suspended parts of $F$ can proceed. This is the only mechanism in Orc to block or terminate parts of a computation.

In contrast to sequential composition, the following expression calls email at most once.

$\operatorname{email}(a, x)<x<(\operatorname{CNN}(d) \quad \mid \operatorname{BBC}(d))$

The pruning combinator is left associative, i.e. $F<\mathrm{x}<G<\mathrm{y}<H$ is $(F<\mathrm{x}<G)<\mathrm{y}<H$. When $\mathrm{x}$ is not used in $F$, one may use the short-hand $F<<$ for $F<x<G$.

The pruning combinator introduces eager concurrent evaluation. Later, we will see that expressions in the Orc language are often converted to pure Orc calculus using the pruning combinator; this introduces concurrency, even in the evaluation of arithmetic expressions, without programmer intervention.

\subsection{Algebraic Properties of the Combinators}

An operational semantics of Orc based on a labeled transition system appears in [18]. Employing bisimulation, we have proven the following algebraic properties of the combinators, some of which resemble laws of Kleene algebra (see [19] for these proofs). Below, we write " $f$ is $x$-free" to mean that $x$ does not occur as a free variable in $f$.

$$
\begin{aligned}
& \text { (Unit of } \mid \text { ) } \\
& \text { (Commutativity of } \mid \text { ) } \\
& f \mid \mathbf{s t o p}=f \\
& f|g=g| f \\
& \text { (Associativity of } \mid \text { ) } \\
& (f \mid g)|h=f|(g \mid h) \\
& \text { (Left zero of } \gg \text { ) } \\
& \text { (Left unit of } \gg \text { ) } \\
& \text { (Right unit of } \gg \text { ) } \\
& \text { (Associativity of } \gg \text { ) } \\
& \text { stop }>x>f=\text { stop } \\
& \text { signal } \gg f=f \\
& f>x>\operatorname{let}(x)=f \\
& (f>x>g)>y>h=f>x>(g>y>h), \\
& \text { if } h \text { is } x \text {-free } \\
& \text { (Distributivity of | over } \gg \text { ) } \\
& (f \mid g)>x>h=(f>x>h \mid g>x>h) \\
& \text { (Right unit of } \ll \text { ) } \\
& f \ll \mathbf{s t o p}=f \\
& \text { (Commutativity of } \mid \text { with } \ll) \quad(f \mid g)<x<h=(f<x<h) \mid g \text {, } \\
& \text { if } g \text { is } x \text {-free } \\
& \text { (Commutativity of } \gg \text { with } \ll) \quad(f>y>g)<x<h=(f<x<h)>y>g, \\
& \text { if } g \text { is } x \text {-free } \\
& \text { (Commutativity of } \ll \text { with } \ll) \quad((f<x<g)<y<h)=((f<y<h)<x<g) \text {, } \\
& \text { if } g \text { is } y \text {-free and } h \text { is } x \text {-free }
\end{aligned}
$$

We can prove, for example, that $(f<x<g)=f \mid($ stop $<x<g)$, if $f$ is $x$-free. This follows from unit of $\mid$, commutativity of $\mid$, and commutativity of $\mid$ over $\ll$. 


\subsection{Definitions}

An Orc expression may be preceded by a sequence of definitions of the form:

$\operatorname{def} E(\bar{x})=F$

This defines a function named $E$ whose formal parameter list is $\bar{x}$ and body is expression $F$. Definitions may be recursive.

A call $E(\bar{p})$ executes the body $F$ with the actual parameters $\bar{p}$ substituted for the formal parameters $\bar{x}$. A function call may publish more than one value; it publishes every value published by the execution of $F$. If multiple concurrent calls are made to a function $E$, all instances of the body $F$ execute concurrently.

Unlike a site call, a function call does not require all of its arguments to have values. Suppose $E$ is called when an actual parameter $q$, corresponding to a formal parameter $y$, does not have a value. As in the pruning combinator, the executions of parts of $F$ that do not depend on $y$ may proceed, and the parts that depend on $y$ will block until $q$ has a value, which is then substituted for $y$.

\section{The Orc Programming Language}

In the preceding section, we introduced a small concurrency calculus, which serves well as a formal model, but is not a practical language for writing larger programs. Now we describe a language by introducing constructs familiar from functional programming. We show how each construct can be represented in the Orc calculus, so that every program can be translated directly into an equivalent expression in the calculus that uses a small set of primitive sites for arithmetic or data structuring operations. We conclude with an example program and its translation into the calculus. For the details of the full language, see the Orc User Guide [9].

\subsection{Functional Aspects of the Language}

\section{Values and Operators}

The Orc language has three types of constants: numbers $(5,-1,2.71828, \cdots)$, strings ("orc", "ceci n'est pas une |", ..), and booleans (true and false). It provides typical arithmetic $(+-\star / \cdots)$, logical $(\& \&|| \cdots)$, and comparison $(=<>\cdots)$ operators. They are written infix with Java-like operator precedence. Parentheses can be used to override this precedence.

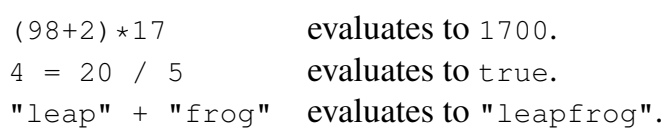


The arithmetic, logical, and comparison operators translate directly to site calls; for example, $2+3$ translates to add $(2,3)$, where add is simply a site that performs addition. A value $\mathrm{v}$ which occurs as an expression on its own becomes a site call let $(\mathrm{v})$.

\section{Nested Expressions and Implicit Concurrency}

The Orc language allows nested expressions, such as $2+(3+4)$. However, $2+(3+4)$ cannot be translated directly to add $(2, \operatorname{add}(3,4))$ as described above; the Orc calculus does not allow expressions, such as add $(3,4)$, to appear as arguments. Instead, we use a fresh variable $\mathrm{z}$ as the argument, and then use a pruning combinator to bind the result of add $(3,4)$ to $z$. Thus the expression $2+(3+4)$ translates to add $(2, z)<z<\operatorname{add}(3,4)$.

Any expression may be nested in this way, even expressions using concurrency combinators. For example, we allow the expression $2+(3 \mid 4)$; it translates to add $(2, z)<z<(3 \mid 4)$. Since the pruning combinator $<z<$ binds only the first value published by $3 \quad 4$ to $z$, the expression could evaluate to either 5 or 6. Furthermore any depth of nesting is allowed, and unfolded in the same way; $2+(3+(4+5))$ becomes add $(2, z)<z<\operatorname{add}(3, y)<y<\operatorname{add}(4,5)$.

This is the fundamental link between Orc as a concurrency calculus and Orc as a functional concurrent language. Since we use the pruning combinator in this translation, all subexpressions are executed concurrently, providing massive implicit parallelism without any additional work by the programmer. See sections 3.2 and 4.1 for examples.

\section{Conditionals}

A conditional expression is of the form if $E$ then $F$ else $G$. If $E$ evaluates to true, then $F$ is evaluated. If $E$ evaluates to false, then $G$ is evaluated. If $E$ does not publish a value, neither $F$ nor $G$ is evaluated.

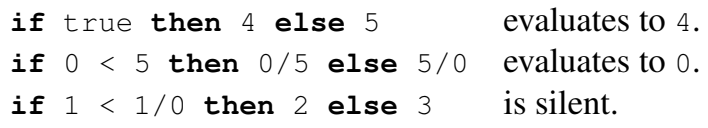

The conditional expression if $E$ then $F$ else $G$ translates to:

( if (b) $>F^{\prime} \mid \operatorname{not}(\mathrm{b})>\mathrm{c}>$ if $\left.(\mathrm{c})>>G^{\prime}\right)<\mathrm{b}<E^{\prime}$

where $E^{\prime}, F^{\prime}$ and $G^{\prime}$ are translations of $E, F$ and $G$, respectively.

Recall that if (true) publishes a signal and if (false) is silent. The site not performs boolean negation.

\section{Variables}

We introduce and bind variables using a val declaration, as follows. Below, $\mathrm{x}$ and $\mathrm{y}$ are bound to 3 and 6 , respectively. 
val $x=1+2$

val $y=x+x$

Variables cannot be reassigned. If the same variable is bound again, subsequent references to that variable will use the new binding, but previous references remain unchanged. Variable bindings obey the rules of lexical scope.

The declaration val $\mathrm{x}=G$, followed by expression $F$, translates to:

$F^{\prime}<\mathrm{x}<G^{\prime}$

where $F^{\prime}$ and $G^{\prime}$ are translations of $F$ and $G$, respectively.

All the rules that apply to the pruning combinator apply to val, and it is permissible to write any Orc expression, even one that publishes multiple values, in a val. One of the most common Orc programming idioms is to write a val to choose the first available publication of a concurrent expression:

val url = Google("search term") | Yahoo("search term")

\section{Data Structures}

The Orc language supports two types of data structures: tuples, such as $(3,7)$ or ("tag", true, false), and finite lists, such as [4,4,1] or ["example"] or []. A tuple or list containing expressions to be evaluated is itself an expression; each of the expressions is evaluated, and the result is a tuple or list of those results.
$[1,2+3]$
evaluates to $[1,5]$.
$(3+4$, if true then "yes" else "no")
evaluates to (7, "yes").

Tuples and lists can contain any value, including other tuples or lists.

The prepend (cons) operation on lists is written $\mathrm{x}: \mathrm{xs}$, where $\mathrm{xs}$ is a list and $\mathrm{x}$ is some element to be prepended to that list.

$[3,5]>t>1: t$ evaluates to $[1,3,5]$.

Data structures are created by site calls. The site let creates tuples directly. The site nil returns the empty list when called. The site cons implements the cons operator and is also used to construct list expressions. For example, [1,2] translates to cons $(1, s)<s<\operatorname{cons}(2, t)<t<\operatorname{nil}()$.

\section{Patterns}

We can bind parts of data structures to variables using patterns. We write _ for the wildcard pattern.

Patterns may replace variables in the $>x>$ and $<x<$ combinators. If a publication does not match the pattern of $a>x>$ combinator, the publication is ignored, and no new instance of the right hand expression is executed. For the $<x<$ combinator, the publication is ignored, and the right hand expression continues to run. 


$$
\begin{array}{ll}
(3,4)>(\mathrm{x}, \mathrm{y})>\mathrm{x}+\mathrm{y} & \text { publishes } 7 . \\
\mathrm{x}<(0, \mathrm{x})<((1,0) \quad(0,1)) & \text { publishes } 1 .
\end{array}
$$

Since the val declaration is simply a different form of the $<x<$ combinator, patterns may replace variables in val as well:

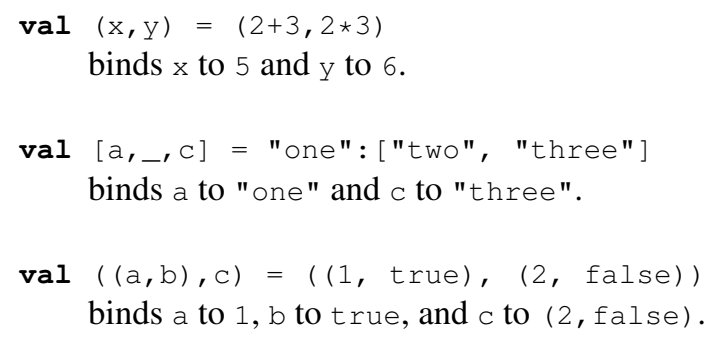

Patterns can be translated into a set of calls to pattern deconstruction sites followed by a set of variable bindings to match up each of the pieces with the appropriate variable names.

\section{Functions}

Functions are defined using the keyword def, and are identical to definitions in the Orc calculus. Definitions may be recursive, and groups of definitions may be mutually recursive.

def $\operatorname{sumto}(\mathrm{n})=$ if $\mathrm{n}<=0$ then 0 else $\mathrm{n}+\operatorname{sumto}(\mathrm{n}-1)$

Functions can be defined as a series of clauses, each of which has a different list of patterns for its formal parameters. When such a function is called, the function body used for the call is that of the first clause whose formal parameter patterns match the actual parameters.

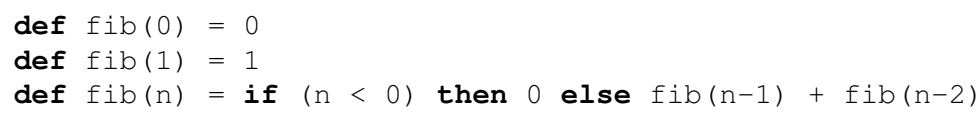

The function fib may also be written more efficiently, as follows:

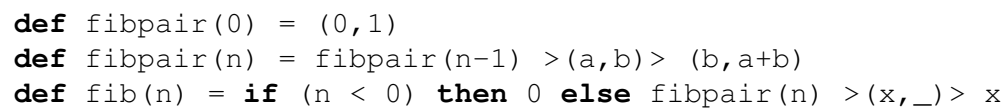

Defining a function creates a value called a lexical closure; the name of the function is a variable and its bound value is the closure, which records all of the current bindings for free variables in the function body.

Since a closure is a value, it can be passed as an argument to another function, thus allowing us to define higher-order functions. As an example, here is the classic map function; see additional examples in Sections 4.1.3 and 4.3.3.

$\operatorname{def} \operatorname{map}(f,[])=[]$

$\operatorname{def} \operatorname{map}(f, x: x s)=f(x): \operatorname{map}(f, x s)$ 
Note the important distinction between $f$ and $f(x)$; the former is a variable whose bound value is a function (closure), and the latter is a call to that function.

\subsection{Implicit Concurrency: An Example}

We show an Orc program that does not use any of the concurrency combinators explicitly. In fact, the program is entirely functional, with the sole exception of the site call random (6), which returns a random integer between 0 and 5. Yet, each nested expression translates into a use of the pruning combinator, making this program implicitly concurrent without any programmer intervention.

The program runs a series of experiments. Each experiment consists of rolling a pair of dice. An experiment succeeds if the total shown by the two dice is c. The function $\exp (n, c)$ returns the number of successes in $n$ experiments.

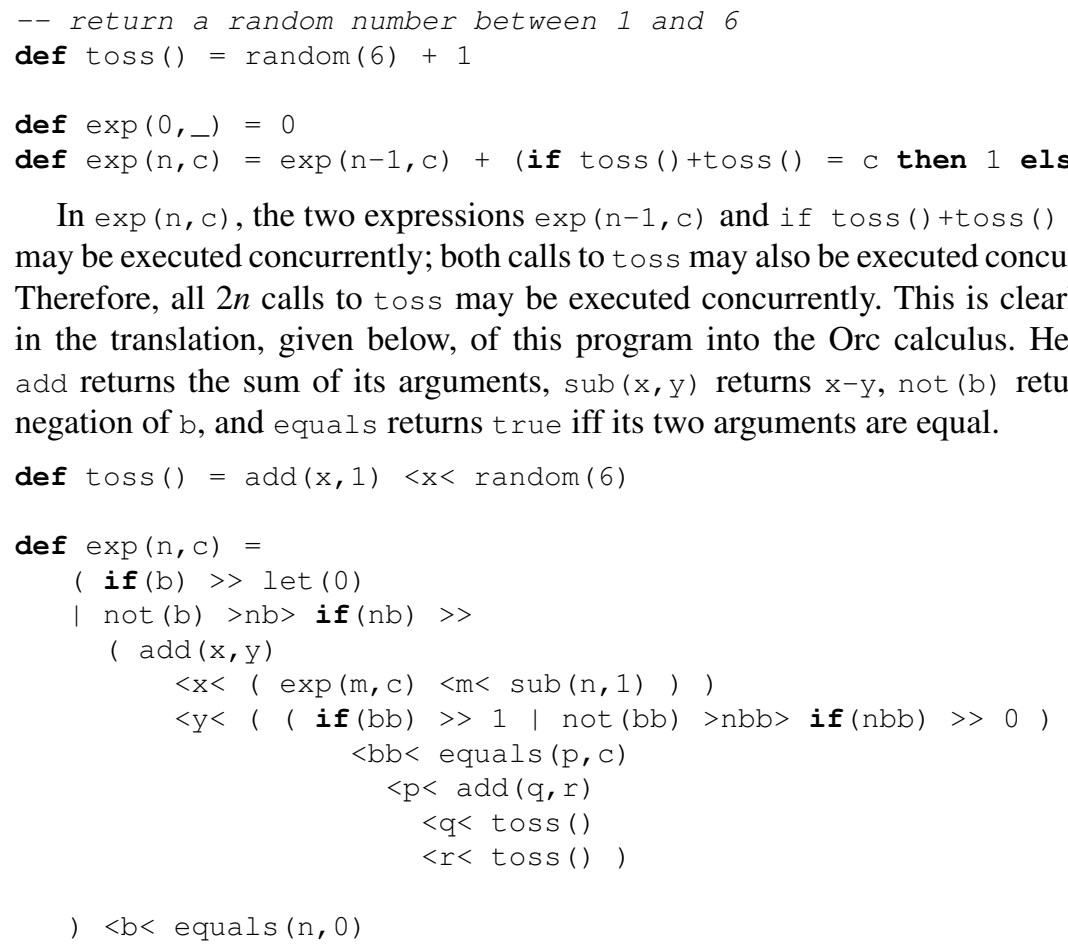

\subsection{Site library}

We have implemented a library of useful sites. We introduce a few essential sites here, and we also note a few properties of sites that were not previously discussed. 
Sites are first-class values

In both the Orc calculus and the Orc programming language, sites are first-class values; they may be bound to variables, passed as arguments, published, and returned by site calls. It is very important that sites can be published by other sites, as this allows the use of "factory" sites, which create new sites such as mutable references or communication channels.

\section{Sites may have methods}

Sites may represent objects with multiple methods, in an object-oriented style. We access methods on sites using a special form of site call, as in c.put (4), which accesses the put method of channel $\mathrm{c}$ and calls it as a site, with argument 4.

This call form, like every other new syntactic form introduced so far, can be encoded in the Orc calculus. The site $c$ is sent a special value called a message, in this case the "put" message. The site responds to that message with another site which will execute the desired method when called. So c.put (4) translates to $c($ put") $>x>x(4)$.

\section{Time}

Orc is designed to communicate with the external world, and one of the most important characteristics of the external world is the passage of time. Orc implicitly accounts for the passage of time by interacting with external services that may take time to respond. However, Orc can also explicitly wait for a specific amount of time, using the special site Rtimer. The call Rtimer $(t)$, where $t$ is an integer, responds with a signal exactly $t$ milliseconds later ${ }^{3}$.

We can use Rtimer together with the $<x<$ combinator to enforce a timeout. Continuing with the example from Section 2.2, we can query BBC for a headline, but allow a default response if $\mathrm{BBC}$ does not respond within 5 seconds.

email(a, $x)<x<(B B C(d) \mid$ Rtimer(5000) $>>$ "BBC timed out.")

\section{References}

Orc does not have mutable variables. Mutable state is provided by sites instead. The Ref site is used to create new mutable references, which are used in a style similar to Standard ML's ref [15].

A call to Ref may include an argument specifying the initial contents of the reference; if none is given, then the reference's value is undefined. Given a reference $r, r$.write $(v)$ overwrites the current value stored in $r$, changing it to $v$, and returns a signal; $r$.read () publishes the current value stored in $r$. If $r$ is undefined, r.read () blocks until a value is written into $r$.

We write $r:=v$ as syntactic sugar for $r$.write (v), and $r$ ? for $r$.read ().

\footnotetext{
${ }^{3}$ An implementation can only approximate this guarantee.
} 


\section{Arrays}

The Array site creates new mutable arrays. Calling Array $(n)$, where $n$ is the size of the array to be created, returns an array a with indices 0 through $n-1$, where the element values are undefined. Elements of array a are accessed by a site call, a (i), which returns a reference to the $i$ th element. That reference can then be read or written just like any reference created by Ref. The expression a. length () returns the length of the array.

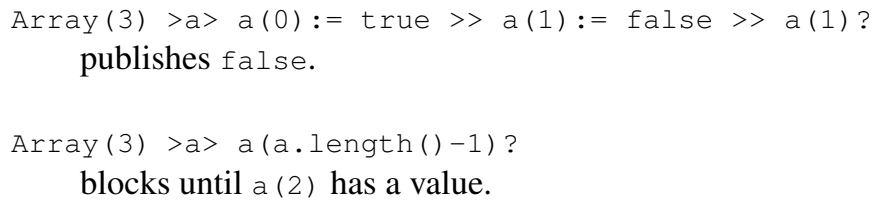

\section{Semaphores}

Unlike other concurrent languages, Orc does not have any built-in locking mechanisms. Instead, it uses the Semaphore site to create semaphores which enable synchronization and mutual exclusion. Semaphore $(\mathrm{k})$ creates a semaphore with the initial value $k$ (i.e. it may be acquired by up to $k$ parties simultaneously). Given a semaphore s, s.acquire () attempts to acquire s, reducing its value by one if it is positive, or blocking if its value is zero. The call s.release () releases s, increasing its value by one. The implementation of semaphore guarantees strong fairness, i.e. if the semaphore value is infinitely often nonzero, then every call to acquire will eventually succeed.

We show below a function that returns an array of $\mathrm{n}$ semaphores, each with initial value 0 .

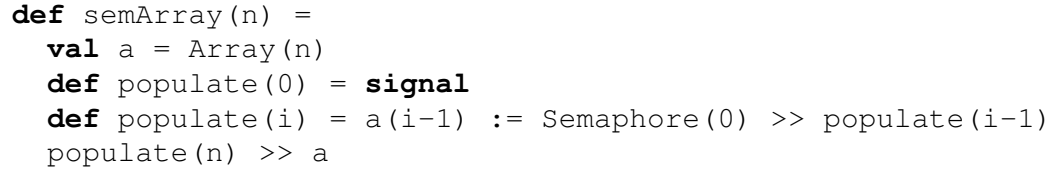

In practice, semaphores and other synchronization sites are only needed when resolving resource conflicts, i.e. concurrent calls to a site that has mutable state. Orc programs with implicit concurrency do not require these arbitration mechanisms.

\section{Channels}

Orc has no communication primitives like $\pi$-calculus channels [13] or Erlang mailboxes [1]. Instead, it makes use of sites to create channels of communication.

The most frequently used of these sites is Buffer, which publishes a new asynchronous FIFO channel. That channel is a site with two methods: get and put. The call c.get () takes the first value from channel $c$ and publishes it, or blocks until a value becomes available. The call $c$.put $(v)$ puts $v$ as the last item of $c$ and publishes a signal. A channel is a value, so it can be passed as an argument. 


\section{Example Programs}

In this section, we present a number of small programs, demonstrating how Orc combines concurrency and recursion, integrates real time, manipulates mutable state, and performs complex synchronizations.

\subsection{Examples using Concurrency and Recursion}

These examples implement some common idioms of concurrent and functional programming. Despite the austerity of Orc's combinators, we are able to encode a variety of idioms concisely.

\subsubsection{Fork-Join}

One of the most common concurrent idioms is a fork-join: evaluate two expressions $F$ and $G$ concurrently and wait for a result from both before proceeding. Thanks to the unfolding of nested expressions described in Section 3.1, this is easily expressed in Orc using just a tuple:

$$
(F, G)
$$

This expands to:

$$
(\mathrm{x}, \mathrm{y})<\mathrm{x}<F<\mathrm{y}<G
$$

We take advantage of the fact that a tuple is constructed by a site call, which must wait for all of its arguments to become available. In fact, any operator or site call may serve to join forked expressions. For example, if $F$ and $G$ each publish a number and we wish to output their maximum value, we simply write $\max (F, G)$, where max returns the maximum of its arguments. We extend this example below.

\section{Simple Parallel Auction}

Orc programs often use fork-join together with recursion to dispatch many tasks in parallel and wait for all of them to complete. Suppose we have a list of bidders in a sealed-bid, single-round auction. Calling b. ask () requests a bid from the bidder $\mathrm{b}$. We want to ask for one bid from each bidder and then publish the highest bid. The function auction performs this task:

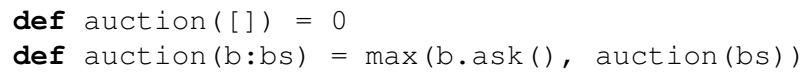

Note that all bidders are called simultaneously. Also note that if some bidder fails to return a bid, then the auction will never complete. Section 4.2.1 presents a different solution that addresses the issue of non-termination by using timeout. 


\subsubsection{Parallel Or}

"Parallel or" is a classic idiom of parallel programming. The "parallel or" operation executes two expressions $F$ and $G$ in parallel, each of which may publish a single boolean, and returns the disjunction of their publications as soon as possible. If one of the expressions publishes true, then the disjunction is true, so it is not necessary to wait for the other expression to publish a value. This holds even if one of the expressions never publishes a value.

The "parallel or" of expressions $F$ and $G$ may be expressed in Orc as follows:

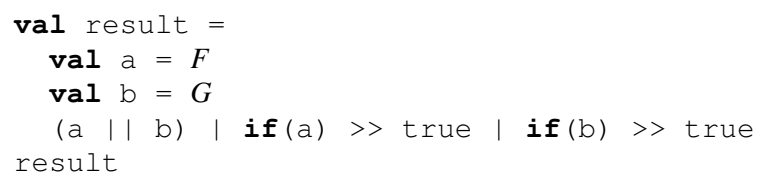

The expression ( $a||$ b) waits for both $a$ and b to become available and then publishes their disjunction. However, if either $a$ or $b$ is true we must publish true immediately, regardless of whether the other variable has a value. Therefore we run if (a) > true and if (b) > true in parallel. Since more than one of these expressions may publish true, we bind only the first result to result. The value of the whole expression is simply the value bound to result.

Note that $F$ and $G$ are evaluated within the binding of result, so that when result becomes bound, $F$ and $G$ are terminated. If val a $=F$ and val b $=G$ were written above val result $=\ldots$, their executions would continue.

\subsubsection{Fold}

We consider various concurrent implementations of the classic "list fold" function, defined by fold $\left(\mathrm{f},\left[x_{1}, \ldots, x_{n}\right]\right)=\mathrm{f}\left(x_{1}, \mathrm{f}\left(x_{2}, \ldots \mathrm{f}\left(x_{n-1}, x_{n}\right) \ldots\right)\right.$. Here is a simple functional implementation:

def fold (_, $[\mathrm{x}])=\mathrm{x}$

def fold $(f, x: x s)=f(x, f o l d(f, x s))$

This is a seedless fold (sometimes called fold1) which requires that the list be nonempty, and uses its first element as the seed. This implementation is shortcircuiting — it may finish early if the reduction operator $f$ does not use its second argument - but it is not concurrent; no two calls to $\mathrm{f}$ can proceed in parallel. However, if $\mathrm{f}$ is associative, we can overcome this restriction and implement fold concurrently. If $\mathrm{f}$ is also commutative, we can further increase concurrency.

\section{Associative Fold}

We define afold $(f, x s)$ where $f$ is an associative binary function and $x s$ is a nonempty list. The implementation iteratively reduces xs to a single value. Each iteration applies the auxiliary function step, which reduces adjacent pairs of items to single values. 


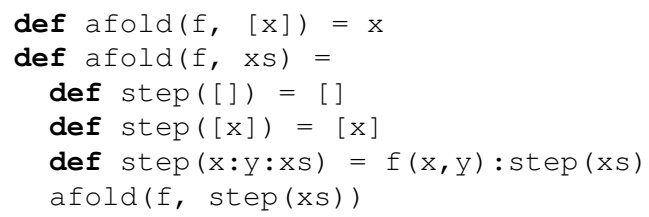

Notice that $f(x, y)$ : step $(x s)$ is an implicit fork-join, as described in Section 4.1.1. Thus, the call $f(x, y)$ executes concurrently with the recursive call step (xs). As a result, all calls to $f$ execute concurrently within each iteration of afold.

\section{Associative, Commutative Fold}

We can make the implementation even more concurrent when the fold operator is both associative and commutative. We define $\mathrm{cfold}(f, x s)$, where $f$ is an associative and commutative binary function and $x \mathrm{~s}$ is a non-empty list. The implementation initially copies all list items into a buffer in arbitrary order using the auxiliary function $x f e r$, counting the total number of items copied. The auxiliary function combine repeatedly pulls pairs of items from the buffer, reduces them, and places the result back in the buffer. Each pair of items is reduced in parallel as they become available. The last item in the buffer is the result of the overall fold.

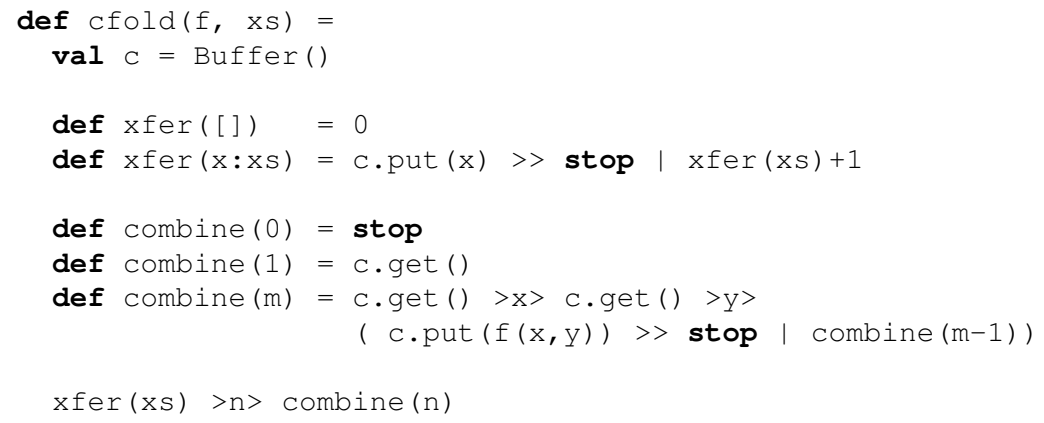

\subsection{Examples using Time}

These examples demonstrate how Orc programs can integrate real time to detect time outs, and execute expressions at regular time intervals, using the site Rtimer described in Section 3.3.

\subsubsection{Timeout}

Timeout, the ability to execute an expression for at most a specified amount of time, is an essential ingredient of fault-tolerant and distributed programming. Orc accom- 
plishes timeout using pruning and the Rtimer site, as we saw in Section 3.3; we further develop that technique in these examples.

\section{Auction with Timeout}

The auction example in Section 4.1.1 may never finish if one of the bidders does not respond. We add a timeout so that each bidder has at most 8 seconds to respond:

def auction([]) = 0

def auction(b:bs) = max(b.ask() | Rtimer(8000) >> 0, auction(bs))

This version of the auction is guaranteed to complete within 8 seconds ${ }^{4}$.

\section{Priority}

We can use Rimer to give a window of priority to one computation over another. In this example, we run expressions $F$ and $G$ concurrently, each of which may publish a single result. For a time interval of one second, $F$ has priority; $F$ 's result is published immediately if it is produced within one second; otherwise, the first value from $F$ or $G$ is published after the time interval.

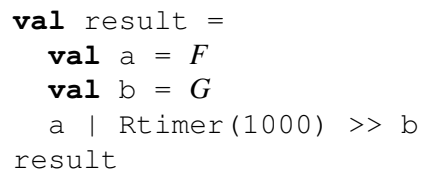

\section{Detecting Timeout}

Sometimes, rather than just yielding a default value, we would like to determine whether an expression has timed out, and if so, perform some other computation. To detect the timeout, we pair the result of the original expression with true and the result of the timer with false. Thus, if the expression does time out, then we can distinguish that case using the boolean value.

Here, we run expression $F$ with a time limit $t$. If it publishes within the time limit, we bind its result to $r$ and execute $G$. Otherwise, we execute $H$.

$\operatorname{val}(r, \mathrm{~b})=(F$, true $) \quad($ Rtimer $(t)$, false)

if b then $G$ else $H$

\subsubsection{Metronome}

A timer can be used to execute an expression repeatedly at regular intervals. We define a function metronome $(t)$, which publishes a signal every t time units.

def metronome $(t)=$ signal $\mid$ Rtimer $(t)>>$ metronome $(t)$

${ }^{4}$ An implementation can only approximate this guarantee. 
The following example publishes "tick" once per second, and "tock" once per second after a half-second delay. The publications alternate: "tick tock tick tock

...". Note that this code is not recursive; the recursion is entirely contained within metronome.

metronome(1000) >> ("tick" | Rtimer(500) >> "tock")

\subsection{Examples using Mutable State}

These examples show how Orc can manipulate mutable state, such as the reference cells and arrays described in Section 3.3. Recall that $\mathrm{x}$ ? is syntactic sugar for $x \cdot \operatorname{read}()$, and $x:=y$ for $x$.write (y). Also recall that the expression a(i) returns a reference to the element of array a at index $i$; array indices start from 0 .

\subsubsection{Simple Swap}

The following function takes two references as arguments, exchanges their values, and returns a signal.

$\operatorname{def} \operatorname{swap}(\mathrm{a}, \mathrm{b})=(\mathrm{a}$ ?, $\mathrm{b}$ ? $)>(\mathrm{x}, \mathrm{y})>(\mathrm{a}:=\mathrm{y}, \mathrm{b}:=\mathrm{x})>>$ signal

\subsubsection{Array Permutation}

The following function randomly permutes the elements of an array in place. It uses the helper function randomize, which for each index $i$ in the array, generates a random number $j$ between 0 and (i-1) inclusive, and swaps a (i-1) with $a(j)$.

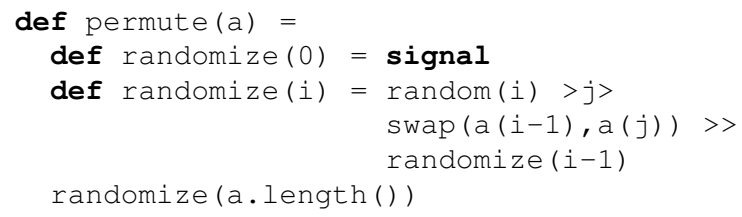

Since random returns values from a uniform distribution, each possible permutation of the array is equally likely. This algorithm originally appears in $[3,10]$.

The technique we use here - traversing an array recursively and calling swap to exchange its elements- is crucial for our Quicksort implementation.

\subsubsection{Applicative Map}

The map function, shown in Section 3.1, applies a function to each element in a list and returns a new list populated with the results; it is a common idiom in pure 
functional programming. When manipulating mutable arrays, it is often helpful to perform a map operation in place: apply the function to each element of the array overwriting the previous contents. The function inplacemap $(f, a)$, defined below, applies the function $f$ to the array a in this way. The helper function mapstep (i) applies $f$ to each element of a with index less than $i$.

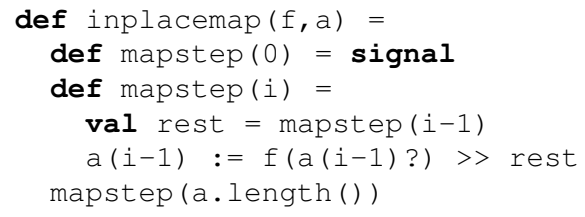

A call to mapstep (i) applies the function $\mathrm{f}$ to element a ( $i-1$ ), and concurrently maps the remainder of the array by calling mapstep (i-1). When mapstep (i-1) completes, rest is bound to a signal, and then mapstep (i) returns.

The following expression increments the value of each element of a by 1 :

$\operatorname{def} \operatorname{inc}(x)=x+1$

inplacemap (inc, a)

\subsection{Examples using Synchronization and Communication}

Synchronization and communication are fundamental to concurrent computing. We implement some examples of synchronization -the rendezvous mechanism [6, 12] and a solution to the readers-writers problem [2]- and show how communicating processes [6] may be programmed in Orc.

\subsubsection{Rendezvous}

The concept of rendezvous between two parties was first introduced by Hoare and Milner as a form of process synchronization. Orc does not include rendezvous as a primitive concept, but we can program it using semaphores. First, we show a twoparty rendezvous, and then generalize it to $(n+1)$-party rendezvous, for $n \geq 1$.

A rendezvous occurs between a sender and a receiver when both of them are waiting to perform their respective operations. They each wait until they complete the rendezvous, and then they can proceed with their computations. A rendezvous involves synchronization and data transfer. In the solution below, first we show only the synchronization, and later data transfer. Potentially many senders and receivers may simultaneously wait to rendezvous, but each can rendezvous with exactly one other party.

Senders and receivers call the functions send and receive, respectively, when they are ready to rendezvous. The solution employs two semaphores, up and down, which are acquired and released in a symmetric manner. (The roles of sender and receiver are symmetric; so the two function bodies may be exchanged.) It can be 
shown that this solution synchronizes a pair of sender and receiver, each of them receives a signal following a synchronization, and that it leaves the semaphores in their original states (with value 0) following the synchronization. We expect each semaphore to be binary-valued, yet this is not a requirement. For general semaphores, there is still pairwise synchronization, though it cannot be ascertained which sender has synchronized with which receiver.

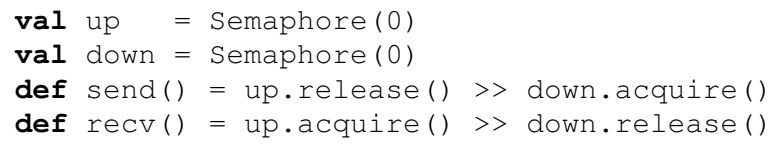

In order for the sender to send data value $\mathrm{v}$, replace semaphore up by buffer $\mathrm{b}$, and modify the programs:

$\operatorname{def}$ send $(\mathrm{v})=\mathrm{b} \cdot \operatorname{put}(\mathrm{v})>>$ down. acquire ()
def receive() $=\mathrm{b} \cdot \operatorname{get}()>\mathrm{x}>$ down.release() $>\mathrm{x}$

The given solution can be generalized to the case where the senders and receivers belong to specific groups, and a rendezvous occurs only between members of a group. In that case, each group uses its own pair of semaphores and corresponding definitions.

\section{$(n+1)$-party Rendezvous}

We generalize the rendezvous algorithm given above to synchronize $n+1$ parties (processes), $n \geq 1$, using $2 n$ semaphores. We create two arrays of semaphores, up and down, using the semArray function defined in Section 3.3. The algorithm is reminiscent of 2-phase commit protocol in databases. Each of the $n+1$ parties calls a function when it is ready to synchronize, like the sender and the receiver above. The process with index $n$ is designated the coordinator, and it calls function coord; all others call ready. Function call ready (i), where $0 \leq i<n$, first releases semaphore up (i) and then waits to acquire down (i). Function coord first acquires all the up semaphores and then releases all the down semaphores. The 2-party rendezvous is a special case where the receiver played the role of the coordinator.

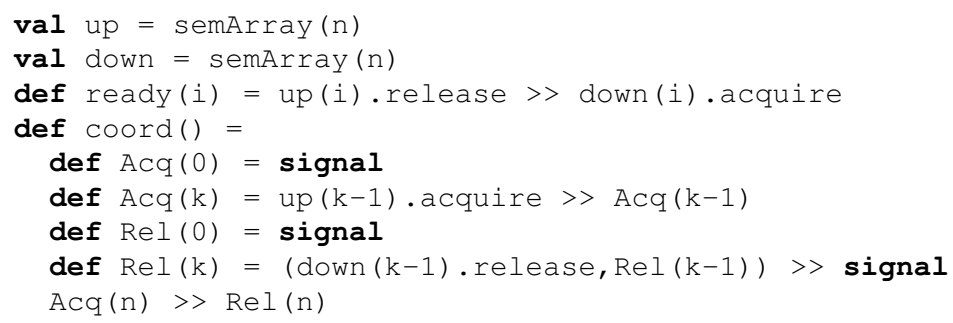




\subsubsection{Readers-Writers Synchronization}

We present a solution to the classical Readers-Writers synchronization problem [2]. Processes, called readers and writers, share a resource such that concurrent reading is permitted but a writer needs exclusive access. We present a starvation-free solution consisting of three functions: start, end, and manager. Readers and writers call start, a blocking operation, to request access; readers call start (true) and writers call start (false). Function start publishes a signal when the resource can be granted. Readers and writers call end () to release the resource. Function manager runs concurrently with the rest of the program to grant the requests.

A call to start (b) adds a request to channel q. Function manager reads from $q$, decides when the request can be granted, and then calls back the requester. We employ semaphores for callback. Specifically,

val $q=$ Buffer ()

def $\operatorname{start}(b)=$ Semaphore $(0)>s>q \cdot p u t((b, s))>>$ s.acquire()

Function manager releases $s$ when it can grant the request. Since $s$ has initial value $0, \mathrm{~s}$. acquire () blocks until the request is granted.

To count the number of active readers and writers, we employ a counter $\mathrm{c}$, a mutable object on which three methods are defined: (1) c.inc () adds 1 to the counter value, (2) c.dec () subtracts 1 from the counter value, and (3) c.onzero() sends a signal only when the counter value is zero. The first two are non-blocking operations, and the last one is blocking. Though onzero () sends a signal only when the counter value is zero, the value may be non-zero by the time the recipient receives the signal. There is a weak fairness guarantee: if the counter value remains 0 continuously, a signal is sent to some caller of onzero(). The counter is initially 0 . The site call counter () creates and returns a new counter.

The code for end merely decrements the counter:

val $\mathrm{C}=$ Counter ()

def end ()$=c \cdot \operatorname{dec}()$

The manager is an eternal loop structured as follows:

def manager ()$=$

$q \cdot \operatorname{get}()>(b, s)>$ if $b$ then $\operatorname{read}(s)$ else write $(s)>>$ manager ()

The invariants in each iteration of manager are that (1) there are no current writers, and (2) the counter value is the number of current readers. We use these invariants in the implementations of read and write.

A reader can always be granted permission to execute, from invariant (1). To satisfy invariant (2), the counter value must be incremented.

def $\operatorname{read}(s)=c \cdot i n c()>>s . r e l e a s e()$

A writer can be granted permission only if there are no active readers. To satisfy invariant (1), the execution of write terminates only when there are no active writers.

def write $(\mathrm{s})=$ c.onZero() > c.inc() > s.release() > c.onZero() 
We start execution of an instance of manager by writing:

val _ $=$ manager ()

The Readers-Writers program in its entirety is shown below.

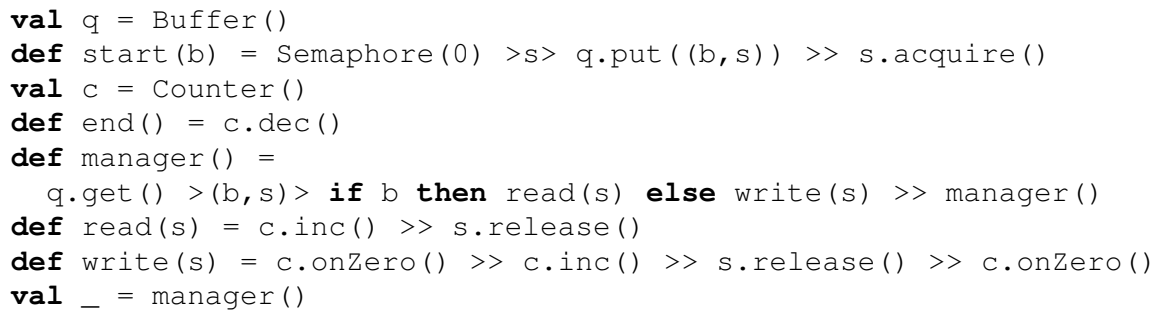

\subsubsection{Process Network}

Process networks [6] and actors $[4,1]$ are popular models for concurrent programming. Their popularity derives from the structure they impose on a concurrent computation. Different aspects of the computation are partitioned among different processes (actors), and the processes communicate through messages over channels. This simple programming model allows a programmer to focus attention on one aspect of a problem at a time, corresponding to a process. Additionally, interference and race conditions among processes, the bane of concurrent programming, are largely eliminated by restricting communication to occur through messages.

We show how this programming model can be incorporated within Orc. Channels are created by calls to Buffer. Processes are represented as Orc definitions, which share these channels. The entire process network is the parallel composition of these processes. Below, we restrict ourselves to FIFO channels though other communication protocols can be defined in Orc.

As an example, consider a transformer process $P$ that reads inputs one at a time from channel in, transforms each input to a single output by calling function (or site) transform, writes the result on channel out, and repeats these steps forever.

$\operatorname{def} P(c, d)=c \cdot g e t()>x>$ transform $(x)>y>d \cdot p u t(y)>P P(c, d)$

$P$ (in, out)

Next, we build a small network of such processes. The network has two processes, and both read from in and write to out.

P(in, out) | P(in, out)

Here, the two processes may operate at arbitrary speeds in removing items from in and writing to out. Therefore, the order of items in the input channel is not necessarily preserved with the corresponding outputs in out. 


\section{Probabilistic Load Balancing}

Consider adding a balancer process that reads from in and randomly assigns the input to one of the processes for transformation, as a form of load balancing. Again, the processes write their results to out, and they may not preserve the input order. We define two internal channels in' and in' ' which link balancer to the transformer processes.

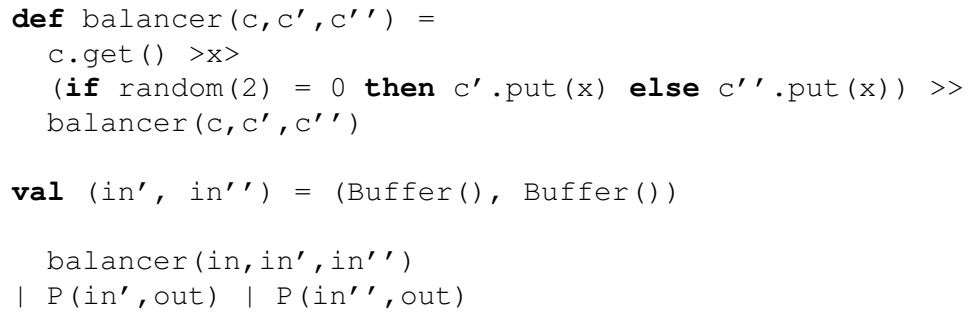

\section{Deterministic Load Balancing}

Now consider a load balancing network in which the order of inputs is preserved in the output. We replace the balancer process with a distributor process that sends alternate input items along $i^{\prime}$ and $i^{\prime \prime}{ }^{\prime}$. The transformer processes write their outputs to two internal channels out' and out' '. And, we add a collector process that copies the values from out' and out' ' alternately to out.

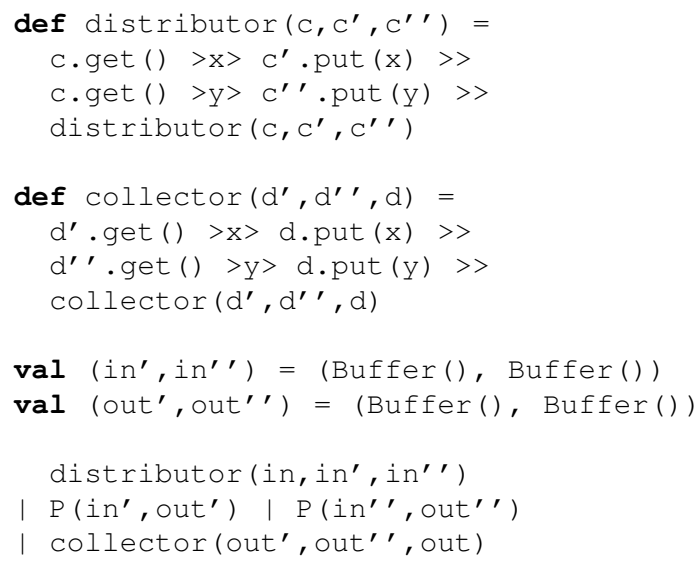

We have shown some very simple networks here; in particular, the networks are acyclic and a-priori bounded in size. See [16] for networks in which arbitrarily many processes are dynamically initiated, interrupted, resumed or terminated. The networks may be structured in a hierarchy where a process itself may be a network to any arbitrary depth, and connections among network components are established statically by naming explicit channels as shown, or dynamically by sending a channel name as a data item. 


\section{Quicksort in Orc}

The Quicksort algorithm focuses on three core ideas in computing: recursion, mutable store, and concurrency. We present an implementation of Quicksort in Orc, in which we show how Orc expresses all three of these ideas. The program is recursive and largely functional in its structure. It uses fork-join when partitioning the array and sorting subarrays, making both the partitioning process and the recursive subsorts implicitly parallel throughout. Furthermore, it manipulates the array elements in place, avoiding the overhead of maintaining extra copies.

We define a quicksort function, which takes as its only argument an array a, and sorts that array in place. When the sort is complete, it returns a signal.

def quicksort $(a)=\ldots$

Within the body of quicksort, we define an auxiliary function part $(p, s, t)$ that partitions the subarray of a defined by indices $s$ through $t$ into two partitions, one containing values $\leq p$ and the other containing values $>p$. One of the partitions may be empty. The call part $(p, s, t)$ returns an index $m$ such that $a(i) \leq p$ for all $s \leq i \leq m$, and $a(j)>p$ for all $m<j \leq t$.

$\operatorname{def} \operatorname{part}(p, s, t)=\ldots$

To create the partitions, part calls two auxiliary functions $\operatorname{lr}$ and $r l$. These functions scan the subarray from the left and right respectively, looking for the first out-of-place element. Function $1 r$ returns the index of the leftmost item that exceeds $\mathrm{p}$, or simply $\mathrm{t}$ if there is none. Function $\mathrm{r} l$ returns the index of the rightmost item that is less than or equal to $p$, or simply $s-1$ if there is none (the value at $a(s-1)$ is assumed to be $\leq \mathrm{p}$ ).

def $\operatorname{lr}(i)=$ if $i<t \& \& a(i) ?<=p$ then $\operatorname{lr}(i+1)$ else $i$

def $r l(i)=$ if $a(i) ?>p$ then $r l(i-1)$ else $i$

Observe that Ir and $r$ may safely be executed concurrently, since they do not modify the array elements.

Once two out-of-place elements have been found, they are swapped using the function swap defined in Section 4.3.1, and then the unscanned portion of the subarray is partitioned further. Partitioning is complete when the entire subarray has been scanned. Here is the body of the part function:

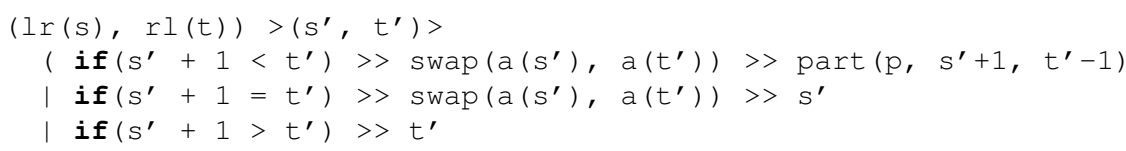

Observe that in the body of part, we use three parallel calls to the if site, with mutually exclusive conditions, each followed by $>>$ and another expression. This is a representation of Dijkstra's guarded commands in Orc, using if to represent a guard, followed by $>>$ and a consequent. Also observe that the second guarded command can be eliminated by replacing the first guard by $s^{\prime}+1<=t^{\prime}$; this incurs a slight performance penalty. 
The main sorting function sort $(s, t)$ sorts the subarray given by indices $s$ through $t$ by calling part to partition the subarray and then recursively sorting the partitions concurrently. It publishes a signal on completion.

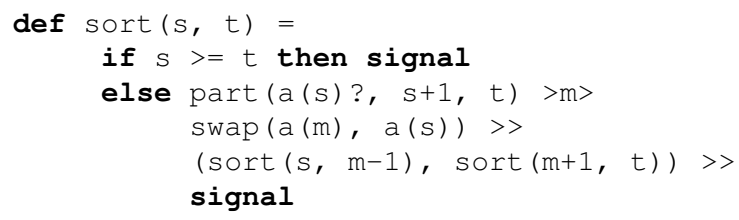

The body of quicksort is just a call to sort, selecting the whole array:

$\operatorname{sort}(0$, a.length ()$-1)$

Here is the quicksort program in its entirety.

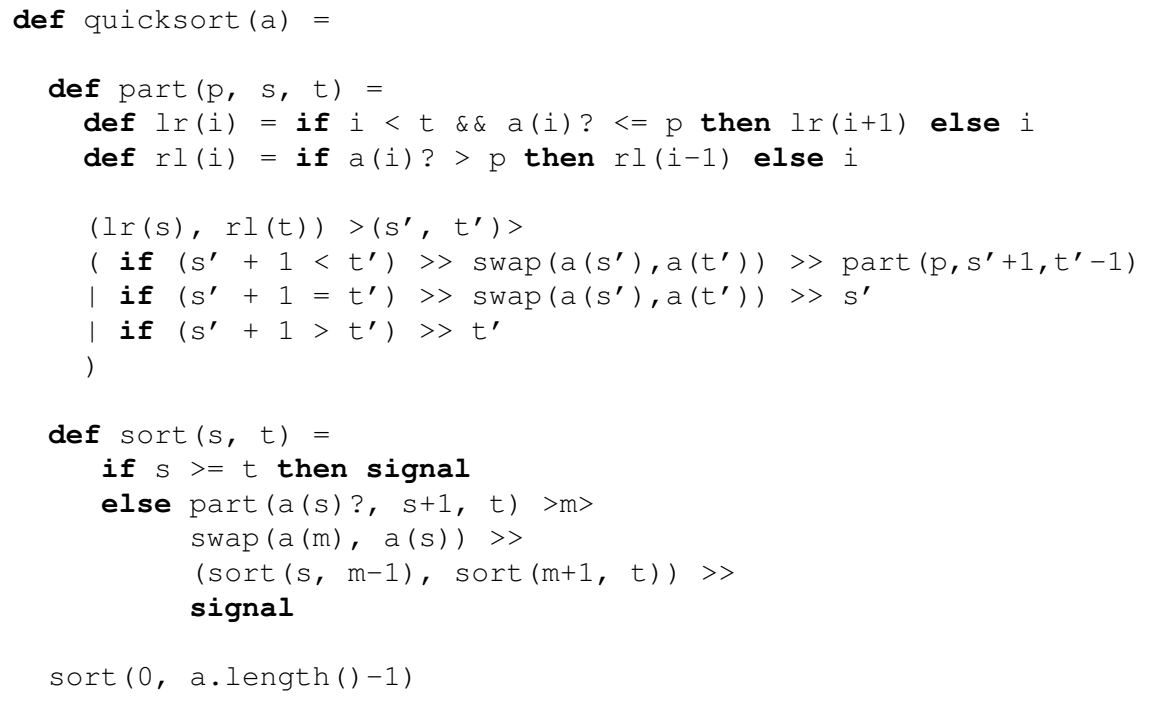

\section{Why Orc?}

Much like other process algebras, the Orc calculus was designed to study the appropriateness of certain combinators for concurrent computing. Unlike most other process algebras, the calculus relies on external sites to deal with non-concurrency issues. Many of the lower-level problems, such as management of locks and shared state, are delegated to sites in Orc. The Orc language was designed to provide a minimal base to experiment with the Orc combinators. Therefore, the language includes only the basic data types and structuring mechanisms. A site library provides additional capabilities for creating references, arrays, and channels, for example. Such a combination has proved fruitful, as we have demonstrated in programming a classic example, Quicksort. 
We hope that designers of future languages will adopt the fundamental principle we have espoused in the design of Orc: seamless integration of concurrency, structure and interaction with the external world.

Acknowledgements Jayadev Misra is deeply grateful to Tony Hoare for research ideas, inspiration and personal friendship spanning over three decades. It is no exaggeration that he would not have pursued certain research directions, that have ultimately proved quite successful, had it not been for Tony's encouragement. This paper is a small token of appreciation.

The authors are indebted to Doug McIlroy and Manuel Serrano for their careful reading of this manuscript and many perceptive comments and suggestions. Our long-time collaborators, Albert Benveniste, Claude Jard, and Jose Meseguer have helped us refine the underlying ideas of Orc.

\section{References}

1. J. Armstrong, R. Virding, C. Wikström, and M. Williams. Concurrent programming in ERLANG (2nd ed.). Prentice Hall International (UK) Ltd., Hertfordshire, UK, UK, 1996.

2. P. J. Courtois, F. Heymans, and D. L. Parnas. Concurrent control with "readers" and "writers". Communications of the ACM, 14(10):667-668, 1971.

3. R. Fisher and F. Yates. Statistical tables for biological, agricultural and medical research. Oliver and Boyd, London, third edition, 1948.

4. C. Hewitt, P. Bishop, and R. Steiger. A universal modular actor formalism for artificial intelligence. International Joint Conference on Artificial Intelligence, 1973.

5. C. A. R. Hoare. Partition: Algorithm 63, quicksort: Algorithm 64, and find: Algorithm 65. Communications of the ACM, 4(7):321-322, 1961.

6. C. A. R. Hoare. Communicating sequential processes. Commun. ACM, 21(8):666-677, 1978.

7. D. Kitchin, W. R. Cook, and J. Misra. A language for task orchestration and its semantic properties. In CONCUR, pages 477-491, 2006.

8. D. Kitchin, A. Quark, W. Cook, and J. Misra. The Orc programming language. In D. Lee, A. Lopes, and A. Poetzsch-Heffter, editors, Formal techniques for Distributed Systems; Proceedings of FMOODS/FORTE, volume 5522 of LNCS, pages 1-25. Springer, 2009.

9. D. Kitchin, A. Quark, W. R. Cook, and J. Misra. Orc user guide. http://orc.csres.utexas.edu/userguide/html/index.html.

10. D. E. Knuth. Seminumerical Algorithms, volume 2 of The Art of Computer Programming. Addison-Wesley, Reading, Massachusetts, third edition, 1997.

11. D. E. Knuth. Sorting and Searching, volume 2 of The Art of Computer Programming. Addison-Wesley, Reading, Massachusetts, second edition, 1998.

12. R. Milner. A Calculus of Communicating Systems. Springer Verlag, 1980.

13. R. Milner. Communicating and Mobile Systems: the $\pi$-Calculus. Cambridge University Press, May 1999.

14. J. Misra. Computation orchestration: A basis for wide-area computing. In M. Broy, editor, Proc. of the NATO Advanced Study Institute, Engineering Theories of Software Intensive Systems, NATO ASI Series, Marktoberdorf, Germany, 2004.

15. L. C. Paulson. ML for the Working Programmer. Cambridge University Press, 1991.

16. A. Quark, D. Kitchin, W. R. Cook, and J. Misra. Orc language project website. http://orc.csres.utexas.edu.

17. R. Sedgewick. Quicksort. PhD thesis, Stanford University, 1975.

18. I. Wehrman, D. Kitchin, W. Cook, and J. Misra. A timed semantics of Orc. Theoretical Computer Science, 402(2-3):234-248, August 2008.

19. I. Wehrman, D. Kitchin, W. R. Cook, and J. Misra. Properties of the timed operational and denotational semantics of Orc. Technical Report TR-07-65, The University of Texas at Austin, Department of Computer Sciences, December 2007. 